\title{
Genetic Algorithm Based on Analytic Hierarchy Process PID Parameter Tuning of UAV Control System
}

\author{
HE Miao-lei, HE Jilin, AO Xiaole \\ The State Key Laboratory of High Performance Complicated Manufacturing, Central South \\ University, Changsha, 410083, China
}

Keywords: Quad-copters; AHP Genetic Algorithms; ID tuning; Multi-objective optimization

\begin{abstract}
The control problems of quad-rotor flight such as under-actuated control, strong coupling were analyzed. With a simplified dynamic model of quad-rotor based on Newton - Euler equation, a double-loop PID controller which consists of the inner attitude control and out position control. Ac cording to the requirements such as static characteristics, dynamic performance and reliability, the $s$ ystem is divided into the system layer, subsystem layer and parameters layer. Aiming the multi-objective optimization index, the method of analytic hierarchy genetic algorithm was used for PID parameters determining. Then outdoor flight experiments were conducted with the controller and parameters obtained. The experimental results show that the aircraft can meet the engineering accuracy requirement of an outdoor flight.
\end{abstract}

\section{Introduction}

The growing interest for the aerial robotics research community in unmanned aerial vehicles (UAV) as exemplified by helicopters [1 4]. Multi-copter is due to the applications in both military scenarios and civil that can be equipped with other sensors to explore building, collect information and investigate dangerous environments. For the reason that light structure, small size, low cost, takeoff and landing model and flexible flight ability, quad-copter research has drawn greater attention. UAV control system is the core technology of the quad-copter system.

Several designs about quad-copter control system in the world, showed in many research papers [5 7]. To realize accurate performance and high dynamic performance, simple, robust and intelligent control laws are introduced in designing the quad-copter control system, for instance, sliding model control, Neural network control, back-step control.

Genetic algorithm (GA) is a kind of intelligent computational model, simulating nature evolutionary mechanism, based on Darwinian Evolution idea. The algorithm starts from random or specific initial solution sets, follows iterative computation logic, such as selection, crossover and mutation operator, calculates and updates new solution set. Based on fittest survive principle, GA leads research result to the optimal solution. Since in nature, fittest individuals dominating over the weaker ones.

A two closed loop PID control system, composed with inner attitude control loop and outer position control loop, using GA to resolve multi-objective optimization problem in control loops is presented in the paper. The control system is applied in an experimental quad-copter, a serial experiment results verify the effectiveness of the proposed algorithm.

\section{Dynamics Model}

\section{Coordinate build}

For the purpose of conveniently describe and dynamics model, the coordinate system is built as Fig.1, body coordinate system and earth coordinate system. Original point of the body coordinate system is set in the center mass of UAV. X-axis is along the vehicles head, Z-axis is at $90^{\circ}$ to the ground, Y-axis is settled following right-hand rule. The two coordinates are overlap completely until the quad-copter takeoff, the earth one is considered as inertial coordinate system, does not change in the UAV flight model. Quad-copter has six degrees of freedom in three-dimensional space. Rotation transfer matrix $\mathrm{R}$ is given by: 


$$
R=\left[\begin{array}{ccc}
C_{\theta} C_{\psi} & S_{\phi} S_{\theta} C_{\psi}-C_{\phi} S_{\psi} & S_{\phi} C_{\theta} S_{\psi}+S_{\theta} S_{\psi} \\
C_{\phi} S_{\psi} & S_{\phi} S_{\theta} S_{\psi}+C_{\theta} C_{\psi} & S_{\phi} C_{\theta} C_{\psi}-S_{\theta} C_{\psi} \\
-S_{\phi} & C_{\phi} S_{\theta} & C_{\phi} C_{\theta}
\end{array}\right]
$$

Where, $\mathrm{C}$ represents cos trigonometric function, and $\mathrm{S}$ represents sin.

\section{Kinetic equation}

The position of the UAV in earth coordinate system is given by $\mathrm{P}=(\mathrm{x}, \mathrm{y}, \mathrm{z}), \quad \phi, \theta$ and $\psi$ represent the roll, pitch and yaw angle. $\Omega=(\mathrm{p}, \mathrm{q}, \mathrm{r})$ is the angular velocity of the vehicle.

The equations are on the condition that the quad-copter is rigid. Follow integration of Newton's second law and Euler equation, consider lift force, gravity, air resistance and centripetal force, quad-copter kinetic equation are given as follows:

$$
\left\{\begin{array}{l}
\dot{P}=V \\
m \dot{V}=F+F_{f}+F_{g} \\
J \Omega+\Omega \times J \Omega=M-M_{f}+M_{c}
\end{array}\right.
$$

Rotation transfer matrix between Euler angle and the angular velocity [14] is given by:

$$
\left[\begin{array}{c}
\dot{\varphi} \\
\dot{\theta} \\
\dot{\psi}
\end{array}\right]=\left[\begin{array}{ccc}
1 & S_{\varphi} T_{\theta} & C_{\varphi} T_{\theta} \\
0 & C_{\varphi} & -S_{\varphi} \\
0 & S_{\varphi} / C_{\theta} & C_{\varphi} / C_{\theta}
\end{array}\right]\left[\begin{array}{c}
p \\
q \\
r
\end{array}\right]
$$

In order to model the quad-copter conveniently, control input parameters is given by $U=(\mathrm{U} 1, \mathrm{U} 2$, U3, U4,), which represents climb, roll, pitch and yaw channels inputs. And other flight models can be decomposed as the four models. $U$ is given by:

$$
U=\left\{\begin{array}{l}
U_{1}=b\left(\omega_{1}^{2}+\omega_{2}^{2}+\omega_{3}^{2}+\omega_{4}^{2}\right) \\
U_{2}=b\left(\omega_{2}^{2}-\omega_{4}^{2}\right) \\
U_{3}=b\left(\omega_{3}^{2}-\omega_{1}^{2}\right) \\
U_{4}=k\left(\omega_{1}^{2}-\omega_{2}^{2}+\omega_{3}^{2}-\omega_{4}^{2}\right)
\end{array}\right.
$$

Kinetic equations of the quad-copter can be calculated, combine equations (1), (2), (3).

$$
\left\{\begin{array}{l}
\ddot{x}=\frac{1}{m}\left(\left(C_{\varphi} S_{\theta} C_{\psi}+S_{\varphi} S_{\psi}\right) U_{1}-K_{1} \dot{x}\right) \\
\ddot{y}=\frac{1}{m}\left(\left(C_{\varphi} S_{\theta} S_{\psi}-S_{\varphi} C_{\psi}\right) U_{1}-K_{2} \dot{y}\right) \\
\ddot{z}=\frac{1}{m}\left(C_{\varphi} C_{\theta} U_{1}-K_{3} \dot{z}-m g\right) \\
\ddot{\varphi}=\frac{1}{I_{1}}\left(L U_{2}-K_{4} L \dot{\varphi}\right) \\
\ddot{\theta}=\frac{1}{I_{2}}\left(L U_{3}-K_{5} L \dot{\theta}\right) \\
\ddot{\psi}=\frac{1}{I_{3}}\left(L U_{4}-K_{6} L \dot{\psi}\right)
\end{array}\right.
$$

\section{Analytic Hierarchy Process}

\section{Flight controller design}

In this section, a new control based on analytic hierarchy and proved by GA PID design method is selected to solve the precise path tracking problem for quad-copter maneuvering flight. Quad-copter is an underactuated control system with six degrees of freedom and four inputs. For the reason that attitude information updating rate is higher than position, the kernel closed loop is settled as attitude control and the outer one is settled as position control. Quad-copter control system control structure is showed as Fig.2. 


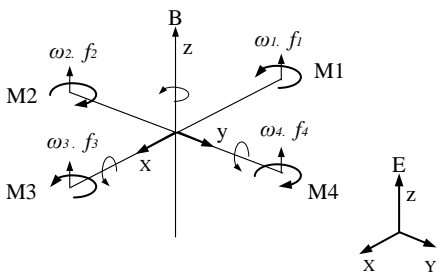

Fig.1. Coordinate System

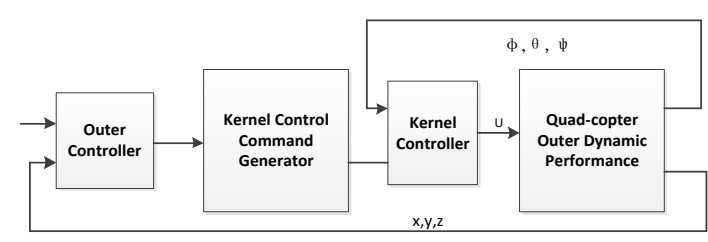

Fig.2. Quad-copter Control System Structure

Proportion Integration Differentiation (PID) control is widely used in different control system, For the reason that PID has the advantages including simple structure, robustness, realized easily, reliable. Quad-copter has six control channels, showed as follows:

Attitude control closed loop:

$\left\{\begin{array}{l}\varphi=k_{p \varphi}\left(\varphi_{d}-\varphi\right)+k_{i \varphi} \int\left(\varphi_{d}-\varphi\right) d_{t}+k_{d \varphi}\left(\dot{\varphi}_{d}-\dot{\varphi}\right) \\ \theta=k_{p \theta}\left(\theta_{d}-\theta\right)+k_{i \theta} \int\left(\theta_{d}-\theta\right) d_{t}+k_{\mathrm{d} \theta}\left(\dot{\theta}_{d}-\dot{\theta}\right) \\ \psi=k_{p \psi}\left(\psi_{d}-\psi\right)+k_{i \psi} \int\left(\psi_{d}-\psi\right) d_{t}+k_{d \psi}\left(\dot{\psi}_{d}-\dot{\psi}\right)\end{array}\right.$

Position control closed loop:

$\left\{\begin{array}{l}\mathrm{x}=k_{p x}\left(x_{d}-x\right)+k_{\mathrm{dx}}\left(\dot{x}_{d}-\dot{x}\right) \\ y=k_{p y}\left(y_{d}-y\right)+k_{d y}\left(\dot{y}_{d}-\dot{y}\right) \\ z=k_{p z}\left(z_{d}-z\right)+k_{d z}\left(\dot{z}_{d}-\dot{z}\right)\end{array}\right.$

\section{GA based on analytic hierarchy process}

GA basic operation include copying, crossing and mutating operation. Copy the individual with high fitness to the new population directly. Cross two random individuals, exchange the chromosome. Mutate random DNA at very low probability. Coping operation is the optimizing capacity of GA, crossing determine the global search ability, mutating makes GA with the ability of random search. GA can realize meta-heuristic global search in solution space, with low reliance on the problem. These characteristics make GA an ideal parameters optimization, apply to complex nonlinear system.

GA objective function is not easy set in face to solve multiple objection optimizing. The ability of steady-state and dynamic characteristics is considered to design the controller in this paper. Quad-copter control system is divided into system level, subsystem and parameter level. Combine hierarchical analysis method and quad-copter system characteristics, different optimization index are proposed. Based on subsystems optimization, weighted optimization index of the system is calculated.

Six control channels are show as equation (5) and (6). Each channel is a subsystem with its own optimization index. For example, attitude closed-loop needs high real-time ability than position closed-loop, while position closed-loop needs high precise ability than attitude closed-loop, different channels have different index.

GA steps are as follows:

Step1: determine the range and code of optimal parameters.

Step2: generate the initial population and determine GA parameters.

Step3: set optimal parameters based on analytic hierarchy process, and calculate population values.

The model of the system optimal is show as Fig.3.

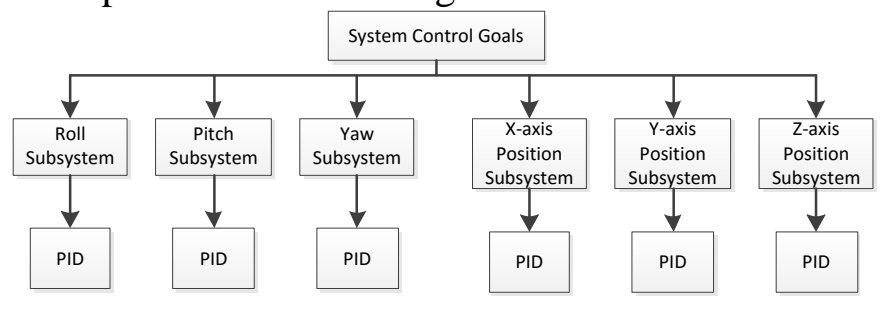

Optimal index is given by:

Fig.3 the System Optimal Model 


$$
\boldsymbol{J}=\sum_{i=1}^{6} \boldsymbol{W}_{\boldsymbol{i}} \boldsymbol{J}_{\boldsymbol{i}}
$$

Where:

$$
J_{i}=\int_{0}^{t}\left(\alpha_{i 1}\left|e_{i}(t)\right|+\alpha_{i 2} u_{i}^{2}(t)\right) d_{t}+\alpha_{i 3} t_{i u}
$$

$\mathrm{W}_{\mathrm{i}}$ is weight value. $J_{1 \ldots} J 6$ represent roll, pitch, yaw, X-axis, Y-axis and Z-axis system optimization index. $W, a_{1}, a_{2}$ and $a_{3}$ are weight values. $J$ is optimal index, $t_{i u}$ is uprising time value. $i$ represents quad-copter six channels.

Step4: coping, crossing, mutating operations

Step5: repeat step3 and step4, until optimal index gets the expected value. Return optimization index and hereditary algebra.

\section{Simulation}

The proposed control strategy has been simulated in order to make sure the quad-copter control system safety and check the performance attained for the path following problem. In the test, with the analysis of the step response diagram, the optimal PID parameters is obtained and solve system optimization problem, using analytic hierarchy process. In the MATLAB GA simulating experiment, sample number Size $=40$, crossover probability $\mathrm{Pc}=0.9$, mutation probability $\mathrm{Pm}=0.05$, gain parameter value range $\mathrm{Kp} \in[0,20], \mathrm{Ki} \in[0,10], \mathrm{Kd} \in[0,10]$. Attitude channel subsystem weight value $\mathrm{W} 1=0.25, \mathrm{~W} 2=0.25$, $\mathrm{W} 3=0.2$. Position channel subsystem weight value $\mathrm{W} 4=0.2, \mathrm{~W} 5=0.1$, W6 $=0.1$. System error $a_{1}=1.0$, controller output weight value $a_{2}=0.1$, uprising time weight value $a_{3}=0.3$. Quad-copter simulation parameters are show in table 1.

\begin{tabular}{|c|c|}
\hline Name (Unit) & Value \\
\hline Vehicle Mass m & 0.6 \\
\hline g & 9.8 \\
\hline K1 K2 K3 & $\begin{array}{lll}0.03 & 0.03 & 0.04\end{array}$ \\
\hline $\mathrm{K}_{4} \mathrm{~K}_{5} \mathrm{~K}_{6}$ & $(6.56,5.56,6.35) \times 0^{-4}$ \\
\hline $\mathrm{I}_{1} \mathrm{I}_{2} \mathrm{I}_{3}$ & $(3.8,3.8,7.1) \times 10^{-3}$ \\
\hline $\begin{array}{c}\text { Blade Distance to } \\
\text { Body Center L }\end{array}$ & 0.2 \\
\hline Lift Coefficient & $3.01 \times 10^{-5}$ \\
\hline $\begin{array}{l}\text { Rotational } \\
\text { Coefficient }\end{array}$ & $3.3 \times 10^{-7}$ \\
\hline
\end{tabular}

Table 1.Quad-copter Parameter

Simulate improved GA in MATLAB to solve parameters optimal problems. In the process, simulation step size is settled as $1 \mathrm{~ms}$, simulating time is $10 \mathrm{~s}$, and hereditary algebra is 60 . The result is show in Fig4. It can be seen that optimal index is nearly best until the algebra is at 30, and the system shows ideal performance. In next section, the controller based on GA using analytic hierarchy process PID parameter tuning is applied in the experimental quad-copter to further verify the comprehensive properties.

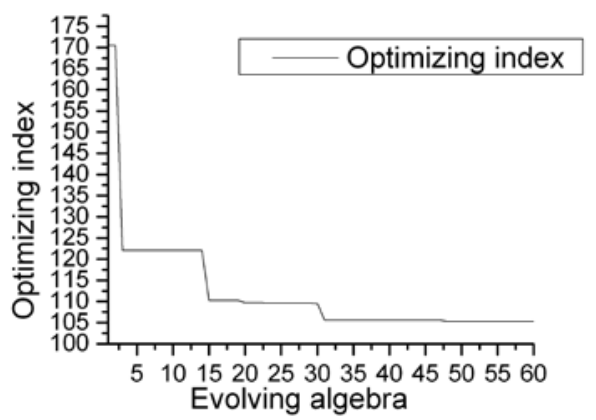

Fig.4 Genetic optimization process 


\section{Experiment}

A flight test has been design to demonstrate the performance of the proposed control. The quad-copter and its flight locus are show in Fig.5. It is a mapping duty about uncharted regions. X, $\mathrm{Y}$ and $\mathrm{Z}$ axis position response is show in Fig.6. The area blew wind with 4 class.in the experiment, GPS and IMU are used to detect position and attitude. Combination GPS and IMU data information is introduced to realize precise position control in order to solve GPS position information low-updating speed problem. The experimental quad-copter takeoffs slowly in hovering flight model until to the design height, then starts scanning mission. From the trajectory of the output variable in Fig.6, we can see that, the GA based on analytic hierarchy process shows efficiency, robustness and high dynamic.

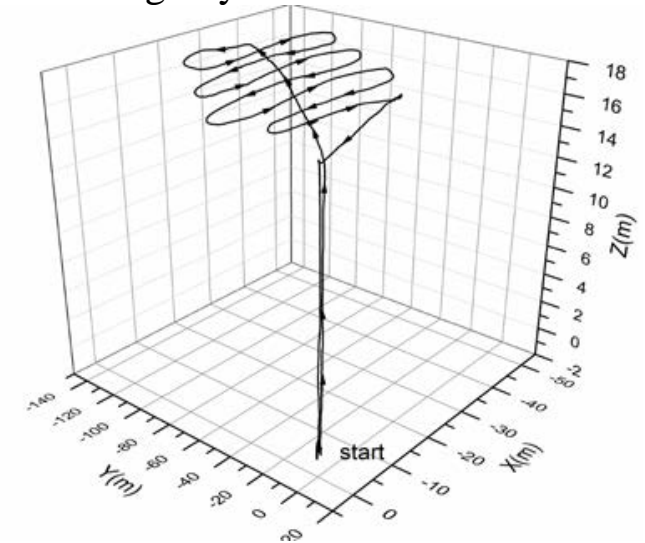

(a)

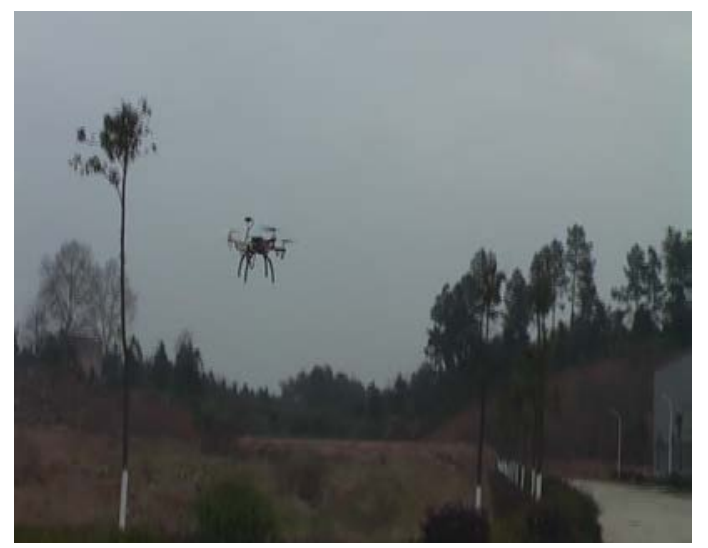

(b)

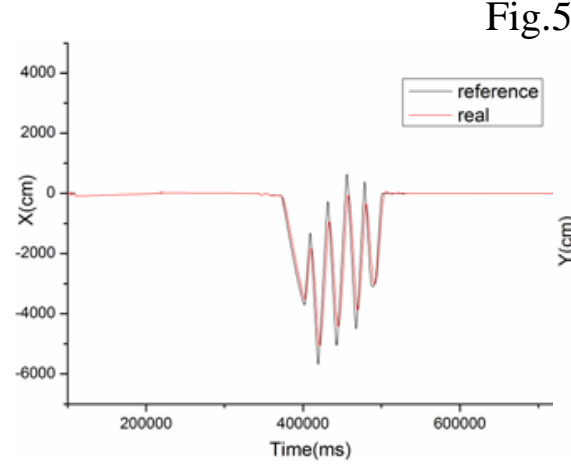

(a)

Fig.5. Absolute Position of the Quad-copter

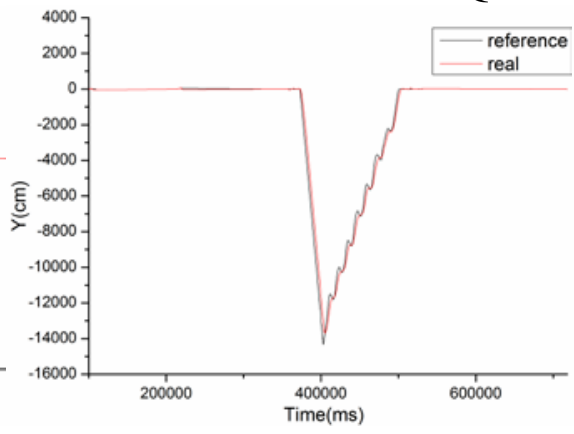

(b)

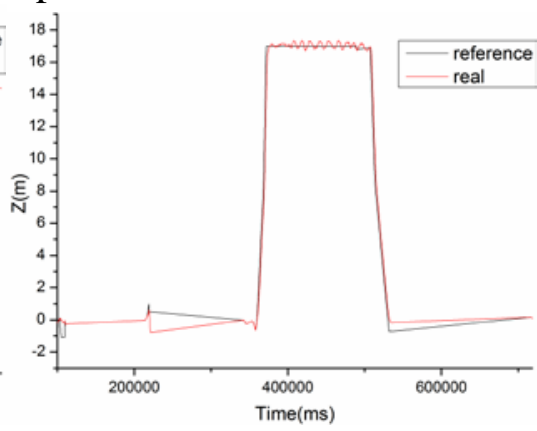

(c)

Fig.6. Trajectory of the output variable

\section{Conclusion}

In this paper, we have presented a new GA control based on analytic hierarchy process and proved with PID method for the position of quad-copter in the presence of external wind disturbance. Dynamic mode is used based on Newton-Euler. The proved PID control is used to overcome the issue of the quad-copter control system. The proposed controller combined with the advantage of PID and proved GA method and compensate the external wind disturbance by using analytic hierarchy process. Simulation and experiments are provided to demonstrate the high precise tracking performance.

\section{Reference}

[1] P Pounds, R Mahony, P Corke. Modelling and control of a large quadrotor robot[J]. Control Engineering Practice, $2010-7$.

[2] J.F.Guerrero-Castellanos,N. Marchand,A. Hably,S.Lesecq,J.Delamare. Bounded attitude control of rigid bodies: Real-time experimentation to a quadrotor mini-helicopter[J].Control 
Engineering Practice . 2011-8.

[3] En-HuiZheng,Jing-JingXiong,Ji-LiangLuo. Second order sliding mode control for aquad-rotor UAV.ISATrans2014;53:1350-6.

[4] Guowei Cai,Ben M. Chen,Tong H. Lee. An overview on development of miniature unmanned rotorcraft systems[J]. Frontiers of Electrical and Electronic Engineering in China. 2010-1.

[5] Abhijit Das, Frank Lewis, Kamesh Subbarao. Backstepping Approach for Controlling a Quadrotor Using Lagrange Form Dynamics[J]. Journal of Intelligent and Robotic Systems . 2009-1.

[6] Luis Rodolfo García Carrillo, Alejandro Enrique Dzul López, Rogelio Lozano, Claude Pégard. Quad rotorcraft control: vision-based hovering and navigation. London: Springer; 2013.

[7] Christos Papachristos, Anthony Tzes. In: 2012 IEEE on modeling and control simulation of an unmanned tilt tri-rotor aerial vehicle. 978-1-4673-0342- 2112/31.00.

[8] Abraham Bachrach,Ruijie He,Nicholas Roy. Autonomous Flight in Unknown Indoor Environments[J]. International Journal of Micro Air Vehicles . 2010-4.

[9] Sergio Salazar-Cruz, Kendoul F, Lozano R, Fantoni I. Real-time stabilization of a small tri-rotor aircraft. IEEE Trans Aerosp Electron Syst 2008; 44(April 2).

[10] Christos Papachristos, Anthony Tzes. In: 2012 IEEE on modeling and control simulation of an unmanned tilt tri-rotor aerial vehicle. 978-1-4673-0342- 2112/31.00. 